\title{
Cuidados com os pés: conhecimento entre pessoas com diabetes mellitus
}

\author{
Feet care: knowledge of people with diabetes mellitus
}

\section{Cuidados con los pies: conocimiento entre personas con diabetes mellitus}

Alice Santos Amaral ${ }^{\mathrm{I}}$, Darlene Mara dos Santos Tavares ${ }^{\mathrm{II}}$

\author{
I Acadêmica do curso de graduação em Enfermagem da Universidade Federal do Triângulo Mineiro (UFTM). Uberaba, MG. E-mail: \\ alicinhaamaral@hotmail.com. \\ II Enfermeira, Doutora em Enfermagem. Docente do curso de graduação em Enfermagem da UFTM. Uberaba, MG. E-mail: \\ darlenetavares@netsite.com.br.
}

\section{RESUMO}

O conhecimento sobre o cuidado com os pés entre as pessoas com diabetes mellitus (DM) contribui para postergar as complicações. Estudo descritivo, prospectivo e transversal realizado em um hospital universitário com o objetivo de descrever as características sócio-demográficas, clínicas e os cuidados com os pés realizados por pessoas com DM. Participaram 58 pessoas, entrevistadas com instrumento semi-estruturado, no período de junho e agosto de 2008 . Os dados foram digitados e analisados no EpiInfo ${ }^{3.2} \mathrm{TM}$. As variáveis categóricas foram submetidas à distribuição de frequência e ao teste qui-quadrado $(p<0,05)$. A maioria é do sexo masculino; está na faixa etária de 60 anos ou mais; possuem $1^{\circ}$ grau incompleto, são casados e aposentados. Metade das pessoas apresenta tempo de diagnóstico inferior a 10 anos, a maioria possui duas co-morbidades; presença de complicações; usam hipoglicemiante oral; não realizam consulta mensal; são cadastrados no Hiperdia; não participam de grupos educativos e seguem o tratamento instituído. Cuidam adequadamente dos pés, exceto a forma como cortam as unhas, o tipo de calçados usados e o ressecamento da pele. Apesar das complicações nos pés em pessoas com diabetes ser prevenível por meio de ações de baixa complexidade, ainda é um desafio para os serviços de saúde.

Descritores: Diabetes mellitus; Pé diabético; Educação em Saúde; Enfermagem; Cuidados de enfermagem.

\section{ABSTRACT}

The knowledge of foot care among people with diabetes mellitus (DM) contributes to delay the complications. Descriptive study, prospective and cross held in a university hospital in order to describe the socio-demographic characteristics, clinics and care with their feet made by people with diabetes mellitus (DM) hospitalized. Involving 58 people, interviewed with semi-structured instrument for the period from June to August of 2008. Data were entered and analyzed in EpiInfo3.2 TM. The categorical variables were subjected to the distribution of frequency and the chisquare test $(p<0.05)$. Most respondents are male, at the age of 60 years old or more; have incomplete 1 st degree, are married and retired. Half of the people have time of diagnosis less than 10 years; the majority has two comorbidities, presence of complications, uses oral hypoglycemic; not hold monthly consultations and are registered with Hiperdia; not participate in educational groups and follow the treatment. Proper care of feet, except for the way they cut the nails, the type of footwear used and dry skin. Despite the complications in the feet in people with diabetes are preventable through actions of low complexity, it is still a challenge for health care.

Descriptors: Diabetes mellitus; Diabetic foot; Health Education; Nursing; Nursing care.

\section{RESUMEN}

El conocimiento sobre el cuidado con los pies entre las personas con diabetes mellitus (DM) contribuye a retrasar las complicaciones. Estudio descriptivo, prospectivo y transversal realizado en un hospital universitario con el fin de describir las características socio-demográficas, clínicas y los cuidados con los pies por personas con diabetes mellitus (DM). Participaron 58 personas, entrevistadas con el instrumento semi-estructurado, en el período comprendido entre junio y agosto de 2008. Los datos fueron digitados y analizados en EpiInfo $3.2 \mathrm{TM}$. Las variables categóricas fueron sometidos a la distribución de frecuencia y la prueba de qui-cuadrado $(p<0,05)$. La mayoría de los entrevistados son hombres, con 60 años o más de edad; poseen el primer grado incompleto, son casados y jubilados. La mitad de la población tiene tiempo de diagnóstico de 10 años, la mayoría tiene dos co-morbilidad: la presencia de complicaciones, uso de hipoglucemiantes orales; no hacen consultas mensuales, estén matriculados con HIPERDIA; no participan en grupos educativos y siguen el tratamiento instituido. Cuidan de manera adecuada de los pies, a excepción de la forma de cortar las uñas, el tipo de calzado usado y la piel seca. A pesar de las complicaciones en los pies en personas con diabetes ser prevenible a través de acciones de baja complejidad, se observa que aún es un desafío para la atención de la salud.

Descriptores: Diabetes mellitus; Pie diabético; Educación en Salud; Enfermería; Atención de enfermería. 


\section{INTRODUÇÃO}

Em 2025, estima-se que $5,4 \%$ da população mundial terá o diagnóstico de diabetes mellitus (DM), com maior prevalência nos países em desenvolvimento e na faixa etária de 45 a 64 anos. Atualmente, no Brasil, acredita-se que existam oito milhões de doentes ${ }^{(1)}$.

O DM é classificado como um grupo de doenças metabólicas caracterizado por hiperglicemia resultante de defeitos na secreção ou ação da insulina ou em ambos(2). Esta doença apresenta alta taxa de morbi-mortalitade impactando de forma negativa na qualidade de vida de seus portadores ${ }^{(1)}$.

As complicações crônicas decorrente do DM podem comprometer vários órgãos, inclusive levando à sua falência. Dentre estas morbidades estão a nefropatia, a retinopatia, a neuropatia e as macroangiopatias. Após 15 anos de DM, é possível que 30 a $45 \%$ tenham comprometimento da retina, 10 a $20 \%$ apresentem nefropatia e 10 a $25 \%$ doença cardiovascular(1).

Estes dados denotam o encargo econômico e social, especialmente quando não se obtém o controle metabólico. Ressalta-se que a maior parte dos custos está relacionada às complicações crônicas, que, muitas vezes podem ser evitadas, reduzidas ou postergadas $^{(3)}$.

As úlceras nos pés e, conseqüente, amputações representam uma das principais complicações crônicas, em decorrência de seu fator mutilante, alta taxas de ocupação e permanência hospitalar, repetidas intervenções cirúrgicas e hospitalizações anuais $^{(1)}$. Os dados demonstram que as úlceras nos pés respondem por 50 a $70 \%$ das amputações não traumáticas e que é 15 vezes mais freqüente entre as pessoas com $\mathrm{DM}^{(4)}$.

Os fatores de risco para as úlceras nos pés são: antecedente de úlcera nos pés; amputação nãotraumática; educação em saúde deficiente; neuropatia que leva a insensibilidade e deformidade; calos e lesões não-ulcerativas; uso de calçados inadequados; tabagismo; hipertensão arterial; dislipidemia, baixa acuidade visual e inacessibilidade ao sistema de saúde ${ }^{(4)}$.

Dentre as atribuições do enfermeiro na atenção básica está a realização da consulta de enfermagem, abordando os fatores de risco relacionados ao DM e suas complicações ${ }^{(4)}$. Neste momento de interação entre o profissional de saúde e a pessoa com DM deve ser realizado o exame físico dos pés visando a prevenção de úlceras no local. Entretanto, tem-se observado na prática profissional que o enfermeiro perde esta oportunidade, por vários motivos, entre eles, falta de infra-estrutura, desconhecimento, demanda reprimida, dentre outros.

Outra estratégia a ser desenvolvida, no referido espaço de trabalho, é a atividade educativa, em que o enfermeiro e a equipe multiprofissional devem estimular o autocuidado com os pés e identificar possíveis fatores de risco para o desenvolvimento das úlceras nos pés. Desta forma, estará favorecida a discussão de ações preventivas com as pessoas com DM e seus familiares ou cuidadores.

A educação em saúde requer tempo, espaço, planejamento, material didático e profissionais capacitados. As estratégias devem ter caráter participativo, pois as ações educativas influenciam no estilo de vida, melhoram os ambientes social e físico e a relação interpessoal entre o profissional e o cliente. Além disso, a educação em saúde, baseada no diálogo, ou seja, na troca de saberes, favorece a compreensão dessa relação no processo saúdedoença e, respectivamente, no intercâmbio entre o saber científico e o saber popular ${ }^{(5)}$.

O maior grau de conhecimento sobre a doença e suas complicações melhora a qualidade de vida; reduz o número de crises de hipoglicemia; postergam as complicações; diminuem as internações hospitalares, melhoram o controle metabólico e ocorre maior aceitação da doença( ${ }^{(6)}$.

A utilização de instrumentos que avaliam o conhecimento de pessoas com DM tem-se constituído em importante recurso nas ações educativas, pois permitem conhecer as necessidades de aprendizagem e redirecionar as estratégias de forma a atender as reais necessidades ${ }^{(7)}$.

A mudança no estilo de vida e nas atitudes necessárias ao tratamento e controle do DM, por vezes, é lenta e gradual. A ação educativa é um recurso que contribui com as mudanças paulatinamente e se constitui em uma das funções essenciais do enfermeiro em toda a rede de cuidados progressivos à saúde. Tem-se a finalidade de subsidiar o planejamento de ações educativas, promover a discussão sobre o tema e contribuir com o cuidado oferecido às pessoas com DM, por meio da prevenção das úlceras nos pés. Nesta perspectiva, delinearam-se os objetivos deste estudo, a saber, descrever as características sócio demográficas, clínicas e os cuidados com os pés realizados por pessoas com DM internados em um hospital universitário.

\section{PROCEDIMENTOS METODOLÓGICOS}

Estudo descritivo, prospectivo e transversal realizado nos setores das Clínicas médica e cirúrgica do Hospital de Clínicas (HC) da Universidade Federal do Triângulo Mineiro (UFTM).

A população foi constituída pelas pessoas internadas nos referidos setores do $\mathrm{HC}$, no período de 02/06/2008 a 03/07/2008 e de 05/08/2008 a 06/09/2008. Os critérios de inclusão foram: ter diagnóstico de DM, aceitar participar do estudo, ter 18 anos ou mais e ambos os sexos. Atenderam aos critérios de inclusão 58 pessoas, que foram entrevistadas. 
A coleta dos dados foi realizada nos meses de junho, julho, agosto e setembro, utilizando-se de instrumento semi-estruturado, previamente testado em estudo piloto. Os dados foram coletados nas enfermarias dos respectivos setores.

As variáveis do estudo foram: demográficas sexo (masculino e feminino); faixa etária (elaboradas em intervalos de dez anos), escolaridade (em anos); sociais - estado conjugal (casado ou mora com companheiro; separado, desquitado, divorciado ou viúvo; nunca casou ou morou com companheiro), atividade profissional (sim, não, aposentadoria, pensão, sem renda); clínicas - tempo de diagnóstico de diabetes ( $<10$ anos, 10 a 20 anos, 20 a 30 anos, > 30 anos); número de co-morbidade; presença de complicações; tipo de tratamento para diabetes mellitus (hipoglicemiante oral, insulina, hipoglicemiante oral e insulina, não medicamentoso); consulta mensal; cadastro no Hiperdia, participação em grupos educativos e seguimento do tratamento instituído; cuidados com os pés - tem o hábito de andar descalço; seca bem os pés após o banho; hidrata os pés com cremes ou óleos; como corta as unhas dos pés; apresenta pele ressecada; apresenta calos; apresenta rachadura nos pés; tipo de calçado costuma usar; analise do calçado internamente antes de calçá-lo; uso meias e calçados com boa adaptação; realização de escalda pés; utilização de remédios caseiros ou medicamentos populares para tratar complicações nos pés; tabagismo; avaliação diária dos pés.

Foi construído banco de dados eletrônico no programa EpiInfo 3.2 TM, para digitação e análise dos dados. As variáveis categóricas foram submetidas à distribuição de freqüência simples e a comparação entre as variáveis categóricas foi realizada por meio do teste qui-quadrado. Considerou significativo quando $p<0,05$.

O projeto desta pesquisa foi submetido e aprovado pelo Comitê de Ética em Pesquisa com Seres Humanos da Universidade Federal do Triângulo Mineiro, protocolo $\mathrm{N}^{\circ}$ 1.131. Aos sujeitos, foram apresentados os objetivos, o Termo de Consentimento Livre e Esclarecido e oferecidas as informações pertinentes. Somente após a anuência do entrevistado e assinatura do referido Termo foi conduzida a entrevista.

\section{RESULTADOS E DISCUSSÃo}

Na Tabela 1, a seguir, apresentam-se as variáveis demográficas, sociais e clínicas da população estudada. 
Tabela 1: Distribuição de freqüência das características demográficas, sociais e clínicas de pessoas com DM internadas no HC da UFTM, Uberaba, 2008.

\begin{tabular}{|c|c|c|c|}
\hline \multirow{2}{*}{\multicolumn{2}{|c|}{ Variáveis }} & \multicolumn{2}{|c|}{ Total } \\
\hline & & $\mathbf{N}$ & $\%$ \\
\hline \multirow{4}{*}{ Sexo } & Masculino & 30 & 51,7 \\
\hline & Feminino & 28 & 48,3 \\
\hline & $18 \mid-20$ & 01 & 1,7 \\
\hline & $20 \mid-30$ & 02 & 3,4 \\
\hline \multirow{6}{*}{ Faixa etária (em anos) } & $30 \mid-40$ & 01 & 1,7 \\
\hline & $40 \mid-50$ & 04 & 6,9 \\
\hline & $50 \mid-60$ & 21 & 36,2 \\
\hline & 60 e mais & 29 & 50,0 \\
\hline & Sem escolaridade & 11 & 19,0 \\
\hline & $1^{\circ}$ grau incompleto & 39 & 67,2 \\
\hline \multirow{5}{*}{ Escolaridade } & $1^{\circ}$ grau completo & 03 & 5,2 \\
\hline & $2^{\circ} \mathrm{grau}$ incompleto & 01 & 1,7 \\
\hline & $2^{\circ}$ grau completo & 00 & 0 \\
\hline & $3^{\circ}$ grau completo & 04 & 6,9 \\
\hline & Casado ou mora com companheiro & 41 & 70,7 \\
\hline \multirow[t]{4}{*}{ Estado conjugal } & Separado, divorciado, desquitado ou viúvo & 11 & 12,1 \\
\hline & Nunca casou ou morou com companheiro & 06 & 10,3 \\
\hline & Sim & 10 & 17,2 \\
\hline & Não & 03 & 6,9 \\
\hline \multirow{4}{*}{ Atividade profissional } & Aposentadoria & 28 & 48,3 \\
\hline & Pensão & 09 & 15,5 \\
\hline & Sem renda & 07 & 12,1 \\
\hline & $<10$ anos & 29 & 50,0 \\
\hline \multirow[t]{4}{*}{ Tempo de diagnóstico de diabetes } & $10 \mid-20$ anos & 21 & 36,2 \\
\hline & $20 \mid-30$ anos & 08 & 13,8 \\
\hline & 0 & 01 & 1,7 \\
\hline & 1 & 14 & 24,1 \\
\hline \multirow{4}{*}{ Número de co-morbidades } & 2 & 19 & 32,8 \\
\hline & 3 & 14 & 24,1 \\
\hline & 4 & 07 & 12,1 \\
\hline & 5 & 03 & 5,2 \\
\hline \multirow{3}{*}{ Presença de complicações } & Sim & 36 & 62,1 \\
\hline & Não & 22 & 37,9 \\
\hline & Hipoglicemiante oral & 32 & 55,2 \\
\hline \multirow{3}{*}{ Tipo de tratamento } & Insulina & 16 & 27,6 \\
\hline & Hipoglicemiante oral e insulina & 08 & 13,8 \\
\hline & Não medicamentoso & 02 & 3,4 \\
\hline \multirow{2}{*}{ Consulta mensal } & Sim & 19 & 32,8 \\
\hline & Não & 39 & 67,2 \\
\hline \multirow{2}{*}{ Cadastro no Hiperdia } & Sim & 54 & 93,1 \\
\hline & Não & 04 & 6,9 \\
\hline \multirow{2}{*}{ Participação em grupos educativos } & Sim & 12 & 20,7 \\
\hline & Não & 46 & 73,9 \\
\hline \multirow{2}{*}{ Segmento do tratamento instituído } & Sim & 45 & 77,6 \\
\hline & Não & 13 & 22,4 \\
\hline
\end{tabular}

Na Tabela 1, verifica-se que foram entrevistados 58 sujeitos, dos quais $51,7 \%$ são do sexo masculino e $48,3 \%$ do feminino. Segundo estudo multicêntrico sobre a prevalência do DM no Brasil, não há diferença entre os $\operatorname{sexos}^{(8)}$.

Quanto à idade, destaca-se que metade da população (50\%) possui 60 anos ou mais de idade, Tabela 1. A maior ocorrência de DM em pessoas com esta faixa etária também foi encontrada em outro estudo, como o realizado em Ribeirão Preto, na qual a média de idade foi de $50( \pm 8,5)$ anos $^{(9)}$. Pesquisa multicêntrica sobre a prevalência do DM no Brasil evidenciou que na faixa etária de 60 a 69 anos há 6,5 vezes mais pessoas com esta doença quando comparada à faixa de 30 a $39 \operatorname{anos}^{(8)}$. Por outro lado, ocorre aumento das hospitalizações com a elevação da idade, particularmente a partir dos 45 anos(10). Investigação realizada com idosos no município deste estudo, evidenciou que $21,5 \%$ foram internados no período de doze meses ${ }^{(11)}$. Estes dados denotam o 
desafio a ser enfrentado pelos serviços de saúde no que concerne à reorganização da atenção à saúde tendo como recorte as doenças crônicasdegenerativas e o processo de envelhecimento humano.

No que se refere à escolaridade, Tabela 1 , a maioria $(67,2 \%)$ possui o primeiro grau incompleto, seguidos pelos que nunca estudaram (19\%). A pouca escolaridade tem sido encontrada em outros trabalhos realizados em serviços de saúde, como o realizado em município do interior do Estado de São Paulo em que $87 \%$ das pessoas com diabetes tinham o primeiro grau incompleto( $(9)$ e o da cidade de Rio Branco, Acre em que $40,4 \%$ eram analfabetos ${ }^{(12)}$. A baixa escolaridade entre pessoas com DM constitui fator agravante para o desencadeamento de complicações crônicas, devido ao possível acesso limitado às informações, pouca habilidade com a leitura e a compreensão para o autocuidado.

A maioria dos entrevistados apresenta o estado conjugal como casado ou morando com companheiro $(70,7 \%)$, Tabela 1 . Tal resultado corrobora com estudo realizado em Ribeirão Preto $(73,3 \%)^{(9)}$. Estes dados são favoráveis para o enfermeiro estabelecer a co-responsabilidade no cuidado das pessoas com DM, com os respectivos companheiros. Investigação realizada com os familiares de pessoas com DM evidenciou que $87,5 \%$ relataram que a doença poderia causar algum problema nos pés, destacando as amputações, úlceras, micoses, dificuldade de cicatrização das feridas e infecção(13).

Em relação à atividade profissional, 48,3\% são aposentados e 15,5\% são pensionistas. Estes dados eram esperados, tendo em vista a faixa etária desta população, sendo também obtido em outra pesquisa, na qual $69,3 \%$ são aposentados ou são do $\operatorname{lar}^{(9)}$. Ressalta-se $17,2 \%$ exercem atividade profissional, fato este que precisa ser aprofundado, pois pode interferir na adesão ao tratamento do DM. O exercício da atividade profissional decorre da necessidade financeira ou do interesse em continuar no mercado de trabalho, situação esta que gera satisfação, contribui com a inserção social e não permite o sentimento de inutilidade.

No que concerne ao tempo de diagnóstico de diabetes, Tabela 1, 50\% possuem a doença há menos de 10 anos e 36,2\% entre 10 |- 20 anos. O maior tempo de duração do DM pode indicar mais gravidade da doença com presença de complicações, a exemplo das úlceras nos pés ${ }^{(14)}$. Neste contexto, o enfermeiro deve realizar o exame dos pés em todos os atendimentos, seja na consulta de enfermagem, nas internações hospitalares, nas visitas domiciliares e nas ações educativas. Desta forma, o enfermeiro contribuirá para que pessoa com DM melhore seu autocuidado, postergando e evitando amputações e complicações.
Observa-se na Tabela 1 que, somente, 1,7\% dos entrevistados não possuem co-morbidades. A maioria apresenta duas $(32,8 \%)$, seguidos pelos que referem uma e três, com percentuais iguais (24,1\%). Este estudo não se propôs a investigar quais as comorbidades presentes. Em pesquisa desenvolvida em Campos dos Goytacazes verificou que o DM foi mais prevalente em pessoas com hipertensão arterial, dislipidemia e excesso de peso(15).

As complicações relacionadas ao DM estão presentes em $62 \%$ dos entrevistados, Tabela 1 . As complicações encontradas com mais freqüência são as oftalmológicas, renais, neurológicas e

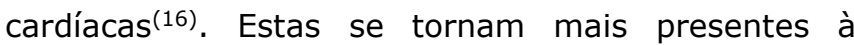
medida que as pessoas têm maior tempo da doença e menor controle metabólico(16).

A presença de mais de uma co-morbidade e de complicações em pessoas com menos de 10 anos de diagnóstico de DM chama a atenção para o atendimento à saúde de pessoas com DM, no sentido do planejamento, organização, acesso e oferta dos serviços de saúde. Se, por um lado, questiona-se como as pessoas com DM estão se cuidando, por outro inquieta-se para o quanto os profissionais, inseridos nos serviços de saúde, têm realizado: ações promocionais de saúde, diagnóstico precoce e atendimento resolutivo.

Quanto ao tipo de tratamento, Tabela 1, a maioria $(55,2 \%)$ faz uso de hipoglicemiante oral, dado este maior do que o encontrado em trabalho $(42 \%)$ realizado em Belo Horizonte ${ }^{(17)}$. Contudo, o uso de insulina $(27,6 \%)$, é menor do que o obtido no referido trabalho (41\%). O uso associado de insulina e hipoglicemiante oral, do presente estudo, representou $13,8 \%$. Percentual alto de pessoas utilizando formas associadas de medicamento denota comprometimento progressivo da doença(9). Ressalta-se que a maior diferença encontrada nesta investigação em comparação às outros é a pequena parcela de pessoas $(3,4 \%)$ utilizando tratamento não medicamentoso. Este percentual representou 24,8\% em estudo realizado em Porto Alegre(16).

O cadastro no Hiperdia foi referido pela maioria $(93,1 \%)$ possui, entretanto, apenas $32,7 \%$ realizam consulta mensal. O Hiperdia é um programa instituído pelo Ministério da Saúde visando à reorganização da atenção à hipertensão arterial e ao diabetes mellitus ${ }^{(4)}$. Realiza o cadastramento e acompanhamento de pessoas com estas doenças e fornece alguns medicamentos. Objetiva definir o perfil epidemiológico dessa população e desenvolver estratégias que visem a modificação do quadro atual e a contribuir com a melhoria da qualidade de vida das pessoas ${ }^{(4)}$.

O baixo percentual de consulta mensal, obtido neste estudo, em relação ao de cadastro no Hiperdia e ao uso de medicamento coloca alguns questionamentos. Por meio da consulta mensal são 
oferecidos os medicamentos e realizado os procedimentos para o acompanhamento e controle do DM. Se os entrevistados não estão comparecendo na consulta mensal, questiona-se como está sendo realizado o acompanhamento da doença e como os medicamentos estão sendo adquiridos. O Ministério da Saúde recomenda que de 60 a $80 \%$ dos casos de DM e hipertensão arterial sejam tratados na rede básica municipal, de forma resolutiva e com qualidade $^{(4)}$. Para o tratamento do DM, são imprescindíveis a vinculação da pessoa às unidades de saúde, a garantia do diagnóstico e o atendimento por profissionais qualificados, uma vez que seu diagnóstico e controle evitam complicações ou, ao menos, retardam a progressão das já existentes ${ }^{(5)}$.

A participação em grupos educativos foi relatada por $20,7 \%$ dos entrevistados, Tabela 1 . Dados semelhantes $(20,8 \%)$ foram encontrados em estudo desenvolvido em Ambulatório de endocrinologia e metabologia, na qual as principais causas da baixa assiduidade foram falta de interesse, horário inadequado, dificuldades no transporte e desconhecimento da existência do grupo(18). Os grupos de educação em saúde fazem parte do tratamento do DM e se constitui em uma forma de abordar aspectos relevantes para o cuidado com esta doença. Nesta perspectiva, questiona-se a baixa adesão e a forma como estão sendo organizadas as ações educativas.

Pesquisa conduzida com pessoas com DM que participam de grupos educativos evidenciou os temas abordados com mais freqüência, a saber, dieta, atividade física e medidas de redução de estresse(19). Os cuidados com os pés não foram citados, ainda que, a literatura científica assinala as úlceras nos pés como complicação prevenível a partir da atuação de equipe multidisciplinar e por ações de baixa complexidade ${ }^{(9)}$.

Verifica-se na Tabela 1 que o tratamento instituído é seguido por 77,6\%. A não adesão ao tratamento, por $22,4 \%$ dos entrevistados é um fator preocupante. É papel do enfermeiro identificar se o tratamento instituído está sendo seguido e a forma como está sendo realizado, para que possa implementar estratégias necessárias à melhor adesão. A falta de adesão pode estar relacionada ao esquecimento ou ao desconhecimento da gravidade da doença, de maneira que, o incentivo para a participação em grupos educativos poderá contribuir para reverter este quadro. Outra investigação mostrou que para $54,7 \%$ dos sujeitos houve diferenças entre a medicação prescrita e a relada(19). Tais dados sugerem dificuldade de adesão ao tratamento prescrito, o que pode aumentar as taxas de morbi-mortalidade, bem como a necessidade de internação hospitalar.

Na Tabela 2, a seguir, apresenta-se as variáveis relacionadas aos cuidados com os pés. 
Tabela 2: Distribuição de freqüência dos cuidados com os pés realizados pelas pessoas com DM internadas no HC da UFTM, Uberaba, 2008.

\begin{tabular}{|c|c|c|c|}
\hline \multirow{2}{*}{ Variáveis } & & \multicolumn{2}{|c|}{ Total } \\
\hline & & $\mathbf{N}$ & $\%$ \\
\hline \multirow{2}{*}{ Tem o hábito de andar descalço } & Sim & 09 & 15,5 \\
\hline & Não & 49 & 84,5 \\
\hline \multirow{2}{*}{ Seca bem os pés após o banho } & Sim & 49 & 84,5 \\
\hline & Não & 09 & 15,5 \\
\hline \multirow{2}{*}{ Hidrata os pés com cremes ou óleos } & Sim & 31 & 53,4 \\
\hline & Não & 27 & 46,6 \\
\hline \multirow{2}{*}{ Como corta as unhas dos pés } & Arredondadas & 41 & 70,7 \\
\hline & Retas & 17 & 29,3 \\
\hline \multirow{2}{*}{ Apresenta pele ressecada } & Sim & 41 & 70,7 \\
\hline & Não & 17 & 29,3 \\
\hline \multirow{2}{*}{ Apresenta calos } & Sim & 09 & 15,5 \\
\hline & Não & 49 & 84,5 \\
\hline \multirow{2}{*}{ Apresenta rachaduras nos pés } & Sim & 29 & 50,0 \\
\hline & Não & 29 & 50,0 \\
\hline \multirow{2}{*}{ Que tipo de calçado costuma usar } & Aberto & 34 & 58,6 \\
\hline & Fechado & 24 & 41,4 \\
\hline \multirow{2}{*}{ Analisa o calçado internamente antes de calçá-lo } & Sim & 42 & 72,4 \\
\hline & Não & 16 & 27,6 \\
\hline \multirow{2}{*}{ Usa meias e calçados com boa adaptação } & Sim & 51 & 87,9 \\
\hline & Não & 07 & 12,1 \\
\hline \multirow{2}{*}{ Faz escalda pés } & Sim & 12 & 20,7 \\
\hline & Não & 46 & 79,3 \\
\hline \multirow{2}{*}{$\begin{array}{l}\text { Utiliza remédios caseiros ou medicamentos populares para tratar } \\
\text { complicações nos pés }\end{array}$} & Sim & 09 & 15,5 \\
\hline & Não & 49 & 84,5 \\
\hline \multirow{2}{*}{ Tabagista } & Sim & 15 & 25,9 \\
\hline & Não & 43 & 74,1 \\
\hline \multirow{2}{*}{ Avalia os pés diariamente } & Sim & 30 & 51,7 \\
\hline & Não & 28 & 48,3 \\
\hline
\end{tabular}

Em relação aos cuidados com os pés, Tabela 2, $15,5 \%$ dos entrevistados tem o hábito de andar descalço. Verificou que há maior proporção de mulheres que andam descalço quando comparado aos homens $\left(X^{2}=5,24 ; p=0,02203028\right)$. Este hábito é prejudicial, principalmente quando associado às deformidades estruturais e perda da sensibilidade dolorosa. O uso de calçados adquire relevância na função de proteger os pés de agentes lesivos externos $^{(9)}$.

Destaca-se na Tabela 2, que 15,5\% das pessoas afirmam não secar bem os pés após o banho. Uma boa higiene dos pés, seguida de secagem adequada, principalmente das regiões interdigitais, é de grande importância para evitar complicações, como as úlceras ${ }^{(12)}$.

O uso de cremes ou óleos para hidratar os pés foi referido por $53,4 \%$ das pessoas entrevistadas, Tabela 2. Manter as pernas e pés hidratados evita o ressecamento da pele e previne o surgimento de lesões. Destaca-se que, proporcionalmente, que as mulheres hidratam mais os pés quando comparado aos homens $\left(x^{2}=25,23 ; p=0,00000051\right)$. Esta situação pode estar relacionada às questões culturais, de maneira que as mulheres cuidam mais de sua pele, buscando postergar o envelhecimento.
Observou também que a escolaridade interfere no cuidado com os pés. Obteve-se maior proporção de pessoas com $2^{\circ}$ e $3^{\circ}$ graus que hidratam os pés quando comparado às com $1^{0}$ grau $\left(X^{2}=12,57\right.$; $p=0,02780948)$. É importante destacar que na realização de atividades de educação em saúde, o enfermeiro deve utilizar linguagem simples, que possa de ser compreendida por todos, independentemente do tempo de estudo.

A maioria $(70,7 \%)$ das pessoas corta as unhas dos pés de forma arredondada, Tabela 2. O corte inadequado das unhas também foi encontrado em estudo realizado no interior de São Paulo, que mostrou um percentual semelhante $(73,3 \%)$ de pessoas com esta prática ${ }^{(9)}$. Tal atitude contribui para o surgimento de lesões nos cantos dos dedos, geradas por encravamento das unhas ou machucados devido ao uso do objeto cortante, que associado à infecção e ao retardo na cicatrização poderá ter a amputação como conseqüência.

O percentual de pessoas com pele ressecada representou $70,7 \%$ e com rachaduras nos pés $50 \%$, Tabela 2. Tais dados estão acima dos encontrados em outro estudo que apresentaram valores de 53,4\% e $29,3 \%$, respectivamente ${ }^{(9)}$. Este fato pode decorrer da presença de complicações crônicas como a 
neuropatia periférica. Ocorre comprometimento das fibras sensitivas, motoras e autonômicas, tendo como conseqüência a redução ou supressão do suor nos pés, deixando-os secos e predispondo-os a rachaduras e fissuras ${ }^{(20)}$.

A presença de calos foi identificada por $15,5 \%$ dos entrevistados, Tabela 2. Estes percentuais estão abaixo dos obtidos em outros estudos que foram de $45 \%{ }^{(17)}$ e $20,2 \%{ }^{(9)}$. As altas pressões em pontos ósseos na região plantar estão associados a calosidades, que são preditores dos processos ulcerativos $^{(20)}$. A comparação entre os sexos denotou que entre os homens há maior proporção que apresentam calos quando comparado às mulheres $\left(X^{2}=4,26 ; p=0,03895948\right)$. É papel do enfermeiro orientar, sensibilizar e motivar as pessoas quanto às mudanças de atitude, que devem incorporar as informações recebidas.

Verifica-se na Tabela 2 que 58,6\% utilizam com mais freqüência sapados abertos. Os calçados abertos, de ponta fina ou sandálias de dedo também foram identificados como a escolha de $41 \%$ de pessoas com $\mathrm{DM}^{(20)}$. Estes dados sugerem 0 desconhecimento das pessoas sobre o risco de lesões com o uso de calçados abertos. Pessoas com DM pode apresentar maior dificuldade de cicatrização, de maneira que tais lesões podem evoluir para amputações. Ao analisar os sexos obteve maior proporção de mulheres usando sapatos abertos quando comparado aos homens $\left(X^{2}=5,88\right.$; $p=0,01527954)$. Mulheres, motivadas pela vaidade, muitas vezes optam pelo uso de sandálias, tanto as do tipo rasteirinha como as com salto, o que pode propiciar o surgimento de lesões nos pés. Indica-se, portanto, o uso de calçados fechados, tipo esportivo ou mocassim, evitando os com costuras internas ${ }^{(9)}$. A escolaridade influenciou no uso de sapatos adequados. Os sapatos fechados são usados em maior proporção por pessoas com mais tempo de estudo ( $2^{\circ}$ e $3^{\circ}$ graus), enquanto que os com menos tempo de estudo (que possuem até o $1^{0} \mathrm{grau}$ ), optam pelos sapatos abertos $\left(x^{2}=12,57\right.$; $p=0,02780948$ ).

De acordo com a Tabela 2, 72,4\% das pessoas analisam o calçado internamente antes de calçá-lo. Esta prática ajuda na prevenção de lesões, uma vez que, o comprometimento do componente sensitivo produz perda gradual da sensibilidade à dor ${ }^{(17)}$. Desta forma, qualquer objeto, ainda que pequeno, presente no interior do sapato pode não ser sentido pela pessoa com DM e causar lesão nos pés.

O uso de meias e calçados com boa adaptação, Tabela 2, foi relatado por $87,9 \%$ das pessoas. Os pontos de alta pressão, calosidades, deformidades nos pés e amputações de dedos são problemas que podem ser evitados com 0 uso de calçados confortáveis ou confeccionados sob medida(20). Os calçados devem ter como principal objetivo proteger os pés de agentes lesivos. No entanto, quando os calçados são extremantes estreitos ou folgados, eles se tornam próprios agentes lesivos. Calçados apertados podem causar lesões no dorso dos dedos e na região lateral do antepé. Por outro lado, calçados folgados podem criar áreas de atrito, que favorecem a formação de bolhas ou, inclusive, o ingresso de pequenos objetos no interior dos mesmos ${ }^{(9)}$. Por isso a importância de calçados que se adaptem bem aos pés.

No que concerne a realização de escalda pés, $20,7 \%$ dos entrevistados a realizam, Tabela 2. A diminuição da sensibilidade em pessoas com DM pode ocasionar queimaduras durante a realização dessa prática, contribuindo para o aumento da úlcera nos pés e, conseqüentemente, elevando o risco de amputação(14). Ao relacionar a participação em grupos educativos e os cuidados com os pés, o estudo mostrou maior proporção de pessoas que participam de grupos educativos que fazem escalda pés quando comparado às que não participam ( $X$ $2=3,99 ; p=0,04583860$ ).

As complicações nos pés são tratadas por 15,5\% das pessoas com remédios caseiros ou medicamentos populares, Tabela 2. Ao analisar a participação em grupos educativos com o tratamento das complicações nos pés, verificou maior proporção de pessoas que participam de grupos educativos utilizando remédios caseiros ou medicamentos populares para tratar complicações nos pés enquanto que os que não participam não realizam tal prática ( $X$ ${ }^{2}=13,49 ; p=0,00024020$ ). Questiona-se, então, mais uma vez, sobre os temas abordados nesses grupos, já que foi observado um resultado significativo de pessoas que os freqüentam e realizam cuidados inadequados com os pés.

O tabagismo foi encontrado em 25,9\% dos entrevistados. Percentuais maiores $(37,6 \%)$ foram encontrados em estudo semelhante ${ }^{(9)}$. O tabagismo é fator de risco podendo causar ou acelerar o processo de aterosclerose ${ }^{(12)}$. O enfermeiro pode aproveitar os momentos de atendimento às pessoas com DM e sensibilizá-los para o abandono dessa prática, evidenciando seus benefícios e malefícios.

Destaca-se na Tabela 2, que $51,7 \%$ dos entrevistados avaliam os pés diariamente. Quando o indivíduo com DM não tem o hábito de inspecionar por completo os pés diariamente, a presença de lesões ou fissuras podem passar despercebidas, até que uma infecção grave tenha se desenvolvido.

É necessário que as pessoas com DM conheçam os mecanismos que podem causar lesões nos pés, para tomarem consciência da necessidade de cuidar de seus pés, tanto com medidas de higiene, hidratação e proteção com calçados apropriados, quanto com a inspeção diária dos pés na procura de algum sinal de lesão, salientando-se a inspeção do interior dos calçados, antes de usá-los ${ }^{(9)}$. 
Nesta pesquisa não foi encontrada diferença significativa entre a idade e os cuidados realizados com os pés. Porém, sabe-se que se trata de um fator que interfere no autocuidado( ${ }^{(9)}$. As lesões de extremidades são mais prevalentes nas faixas etárias mais elevadas e com mais tempo de diagnóstico( ${ }^{(9)}$.

\section{CONSIDERAÇÕES FINAIS}

No presente estudo verificou que a maioria dos entrevistados é do sexo masculino; está na faixa etária de 60 anos ou mais; possuem $1^{\circ}$ grau incompleto, são casados ou moram com companheiro e são aposentados.

Em relação às características clínicas, metade das pessoas apresenta tempo de diagnóstico inferior a 10 anos, a maioria possui duas co-morbidades; presença de complicações; usam hipoglicemiante oral; não realizam consulta mensal; são cadastrados no Hiperdia; não participam de grupos educativos e seguem o tratamento instituído.

Ressalta-se que a maioria cuida adequadamente dos pés, com exceção da forma como corta as unhas, o tipo de calçados que usam e o ressecamento da pele. Há maior proporção de mulheres que andam descalço e hidratam os pés quando comparadas aos homens. Proporcionalmente as pessoas com $2^{\circ}$ e $3^{\circ}$ graus hidratam mais os pés quando comparado às com $1^{\circ}$ grau de escolaridade. Constatou-se que entre os homens há maior proporção que apresentam calos quando comparado às mulheres e que há maior proporção de mulheres usando sapatos abertos quando comparado aos homens. Os sapatos fechados são usados em maior proporção por pessoas com mais tempo de estudo, enquanto que os com menos tempo de estudo optam pelos sapatos abertos. Ao relacionar a participação em grupos educativos e os cuidados com os pés, o estudo mostrou maior proporção de pessoas que participam de grupos educativos que fazem escalda pés e que utilizam remédios caseiros ou medicamentos populares para tratar complicações nos pés quando comparado às que não participam.

A presença de úlceras nos pés e amputações, apesar de preveníveis, ainda é prevalente nos serviços de saúde, levando a danos irreparáveis na vida dessas pessoas. Avaliar o conhecimento acerca dessa e de outras complicações do DM, função do enfermeiro e de toda equipe multidisciplinar, contribui para subsidiar as ações em saúde, em especial, as educativas, visando a promoção da saúde e prevenção de complicações.

\section{REFERÊNCIAS}

1. Ministério da Saúde; Secretaria de Atenção à Saúde; Departamento de Atenção Básica. Diabetes Mellitus. Brasília (Brasil): Ministério da Saúde; 2006.
2. American Diabetes Association. Diagnosis and Classification of Diabetes Mellitus. Diabetes Care. 2008;31 Suppl S55-60.

3. McLellan KCP, Motta DG, Lerario AC, Campino ACC. Custo do atendimento ambulatoriale gasto hospitalar do diabetes mellitus tipo 2. Saúde em Revista. 2006;8(20):37-45.

4. Ministério da Saúde; Secretaria de Políticas Públicas; Departamento de Ações Programáticas Estratégicas. Plano de reorganização da atenção à hipertensão arterial e ao diabetes mellitus. Brasília (Brasil): Ministério da Saúde; 2002.

5. Briceño-Leon R. 1996. Siete tesis sobre la educación sanitária para la participación comunitaria. Cad. Saúde Pub. 1996;12(11):7-30.

6. Maia FFR, Araujo LR. Projeto "Diabetes Weekend" - Proposta de Educação em Diabetes Mellitus Tipo 1. Arq Bras Endócrinol Metab. 2002;46(5):566-73.

7. Cárdenas JMN, Moctezuma RR, Miranda CM, Santiago JLH. Nivel de information médica sobre diabetes, actitud de los pacientes hacia la enfermedad y su asociación com el nivel de control glucémico. Aten Primaria. 2000;26(5):283-6.

8. Malerbi DA, Franco LJ. Multicenter study of the prevalence of diabetes mellitus and impaired glucose tolerance in the urban Brazilian population aged 30 69 yr. Diabetes Care. 1992;15(11):1509-16.

9. Ochoa-Vigo K, Torquato MTCG, Silvério IAS, Queiroz FA, De La Torre Ugarte-Guanilo MC, Pace AE. Caracterização de pessoas com diabetes em unidades de atenção primária e secundária em relação a fatores desencadeantes do pé diabético. Acta paul. enferm. 2006;19(3):296-303.

10. Rosa RS, Schmidt MI, Duncan BB, Souza MFM, Lima AK, Moura L. Internações por diabetes mellitus como diagnóstico principal na Rede Pública do Brasil, 1999-2001. Rev. Bras. Epidemiol. 2007;10(4):46578.

11. Tavares DMS, Guidetti GECB, Saúde MIBM. Características sócio-demográficas, condições de saúde e utilização de serviços de saúde por idosos. Rev. Eletr. Enf. [Internet]. 2008 [cited 2009 dez 30];10(2):299-309. Available from: http://www.fen.ufg.br/revista/v10/n2/v10n2a02.htm

12. Cosson ICO, Oliveira FN, Adan LF. Avaliação do Conhecimento de Medidas Preventivas do Pé Diabético em Pacientes de Rio Branco, Acre. Arq Bras Endocrinol Metab. 2005;49(4):548-56.

13. Pace $A E$, Nunes PD, Ochoa-Vigo $K$. O conhecimento dos familiares acerca da problemática do portador de diabetes mellitus. Rev. Latino-Am. Enfermagem. 2003; 11(3):312-19.

14. Carvalho CBM, Neto RM, Aragão LP, Oliveira MM, Nogueira MB, Forti AC. Pé diabético: análise bacteriológica de 141 casos. Arq Bras Endocrinol Metab. 2004;48(3):398-405. 
15. Souza LJ, Chalita FEB, Reis AFF, Teixeira CL, Gicovate Neto C, Bastos DA, et al. Prevalência de diabetes mellitus e fatores de risco em Campos dos Goytacazes, RJ. Arq Bras Endocrinol Metab. 2003;47(1):69-74.

16. Grillo MFF, Gorini MIPC. Caracterização de pessoas com Diabetes Mellitus Tipo 2. Rev Bras Enferm. 2007;60(1):49-54.

17. Calsolari MR, Castro RF, Maia RM, Maia FCP, Castro $A V$, Reis $R$, et al. Análise retrospectiva dos pés de pacientes diabéticos do ambulatório de diabetes da Santa Casa de Belo Horizonte, MG. Arq Bras Endocrinol Metab. 2002;46(2):173-76.

18. Capilheira MF, Santos IS. Fatores individuais associados à utilização de consultas médicas por adultos. Rev Saúde Pública. 2006;40(3):436-43.

19. Paiva DCP, Bersusa AAS, Escuder MML. Avaliação da assistência ao paciente com diabetes e/ou hipertensão pelo Programa Saúde da Família do município de Francisco Morato, São Paulo, Brasil. Cad. Saúde Pública. 2006;22(2):377-85.

20. Ochoa-Vigo K, Pace AE. Pé diabético: estratégias para prevenção. Acta paul. enferm. 2005;18(1):1009.

Artigo recebido em 18.12.08.

Aprovado para publicação em 26.08.09.

Artigo publicado em 31.12.09. 